\title{
The Effect of ScanSAR Parameters on Interferogram Quality
}

\author{
Ian Cumming and Frank Wong \\ Dept. of Electrical and Computer Engineering \\ The University of British Columbia, Vancouver, BC, Canada V6T 1 Z4. \\ tel: 604-822-4623 fax: 604-822-5949 ianc@ece.ubc.ca
}

\begin{abstract}
When burst-mode data is used for interferometry, the burst cycles must be well-aligned, as well as the normal condition of having the Doppler centroids well-aligned. In this paper, we show the phase error effects of different burst lengths, various burst misalignments, and of receiver noise.
\end{abstract}

\section{Introduction}

With present and future missions such as RADARSAT, ENVISAT and SRTM using ScanSAR to obtain wide swath coverage, there is increased interest in obtaining interferometric products in this mode. Compared to the continuous-mode case, where baseline length and Doppler centroids must be controlled within given limits, the ScanSAR case offers the additional challenge of aligning the burst cycles to obtain acceptable interferometric products.

The effect of burst misalignment has been discussed in [1]. In this paper, we take an experimental approach to investigate how the various ScanSAR parameters interact to affect the quality of interferometric products. Using simulated data, we show how the interferogram phase noise is affected by combinations of:

1. different burst lengths (number of beams),

2. different amounts of burst mis-alignment, and

3. various amounts of receiver noise.

The parameters are chosen to correspond to the typical operating modes of the ENVISAT system. In the various modes, the effect of burst mis-alignment is quantified, and alignment accuracy requirements for acceptable interferometric operation can be inferred.

\footnotetext{
${ }^{1}$ Presented at the 1998 International Geoscience and Remote Sensing Symposium, IGARSS'98, Seattle, July 6-10, 1998. This work is supported by the NSERC/MDA chair in Radar Remote Sensing.
}

\section{Burst-Mode Data}

Burst-mode data is obtained when SAR works in a ScanSAR mode. In this mode, the satellite antenna scans through different range subswaths in order to image a larger range swath. In each subswath, the received range signal is the same as the continuous range signal, however, in the azimuth direction, the data is blocked into bursts.

Figure 1 shows the difference between continuous and burst-mode data. In continuous mode, the Doppler spectrum of each target covers the full range from $f_{1}$ to $f_{2}$. However, in burst mode, each target has a different range of spectral exposure, depending on the target's azimuth location. In Figure 1, Targets $1-5$ are evenly spaced in azimuth, and it can be seen how their spectral signatures vary within the same burst (data block).

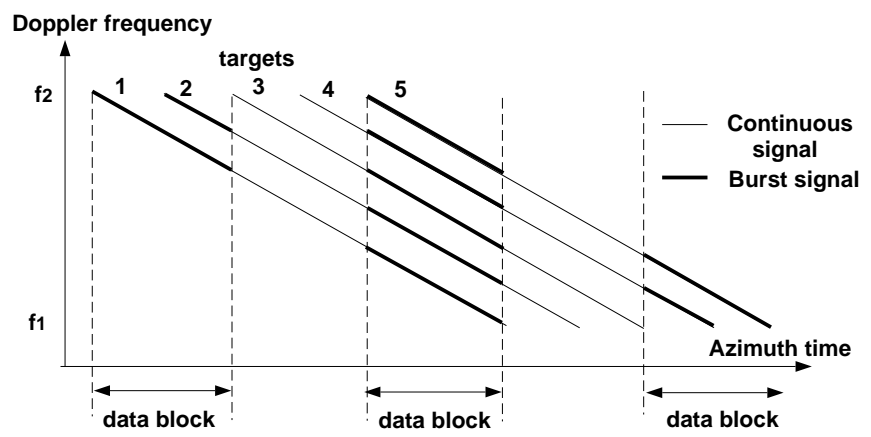

Figure 1: Target spectral distribution in continuous and burst mode

For repeat-pass interferometric applications, the second pass should have the same frequency/time properties as the first pass. For continuous-mode data, this means that the second pass should have the same Doppler centroid as the first pass, so that the same parts of the Doppler spectrum are illuminated in each case. When the data is collected in burstmode, there is the additional requirement that the 
data blocks be aligned in azimuth time, so that the same frequency/time data collection (as illustrated in Figure 1) is obtained.

If the antenna is mis-aligned by a small amount in pass 2 causing a Doppler centroid shift of $\Delta F$ $\mathrm{Hz}$, then a time shift in the burst cycles of $\Delta t=$ $-\Delta F / K a$ s will compensate for the Doppler misalignment for most targets. However, there will be targets for which this antenna mis-alignment will not be compensated, giving them low or zero coherence in the interferogram.

\section{Simulation Methodology}

Our method of simulation has two main steps. First, a real SLC image (the master) and an associated real DEM are used to create a second SLC image (the slave) with the appropriate interferometric phase shift given by the topography and the satellite baseline. Then noise is added to obtain an interferogram with the desired coherence.

The key feature of the simulation is that the master and slave images are convolved with a sinc-like point spread function (PSF) in azimuth, so that each target has the same interferometric phase throughout its PSF (which is a function of the Doppler centroid). This implies the assumption that the original master SLC image represents the ground reflectivity and phase with no point spread function included.

Second, the master and slave SLC images are "uncompressed" into the azimuth time domain, so that the data can be pruned to emulate the burst-mode data collection pattern. After pruning, the data can be re-compressed with a burst-mode SAR processing algorithm [2]. In fact, if this second step is done, the convolving with the PSF which is normally done in the first step can be omitted, as its effect is obtained automatically in the second step by the expansion/compression operation.

A 2-D Gaussian hill was used to show the noise and misalignment effects. Starting from a unit-variance master SLC image $s_{m}(\eta)$, the slave image $s_{s}(\eta)$ is created by:

$$
s_{s}(\eta)=s_{m}(\eta) \exp \left(j \phi_{d e m}\right)+G n(\eta)
$$

where $\eta$ is the azimuth time index, $\phi_{d e m}$ is the phase due to the DEM (with the flat earth fringes removed), $n(\eta)$ is unit-variance random complex Gaus- sian noise, and $G$ is the noise standard deviation. The hill contained 4 phase cycles, and the associated reference interferogram is shown in Figure 2.

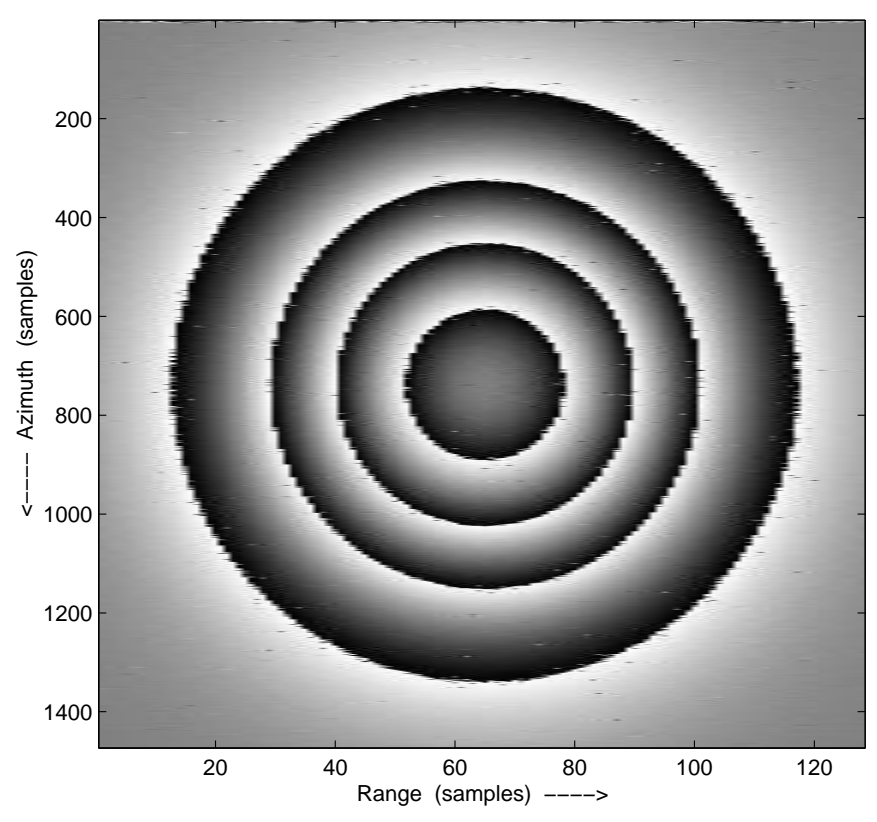

Figure 2: Simulated interferogram of Gaussian hill.

The slave image is then shifted in azimuth time to emulate a burst mis-alignment of a specified number of samples. The master and slave are then expanded with an azimuth chirp, and selected (synchronized) parts of the time-domain array are replaced with zeros to model the missing data of burst-mode operation. The master and slave are then compressed with the SIFFT burst-mode processing algorithm [2]. Then an interferogram is made between the master and slave, and its phase compared with a no-noise, no-shift, continuous-mode interferogram.

\section{Effect of Burst Cycles}

The main effects of the burst cycles are a function of the number of bursts per synthetic aperture, and the "duty cycles" of the bursts. Together these define the resolution per look (as a fraction of the ideal resolution), and the number of looks. The duty cycles are usually governed by the number of ScanSAR beams - if there are 4 beams in operation, the duty cycle is $25 \%$ on any given beam.

In these simulations, we consider the 2- and 4beam cases, with one look taken per aperture. The continuous case is modeled with a 512-point IFFT with no data pruning, the 2-beam case with a 256 - 
point burst and the 4-beam case with 128-point bursts. The simulations are first done with no added noise, then with noise added which gives a continuous-mode coherence of approximately 0.7. The results are shown in Table 1 with $G=0$ and $G=0.25$ - a single look was assumed with no phase smoothing.

The first row of Table 1 represents the ideal case, against which all other cases are compared. In the 2- and 4-beam cases with no noise, some noise enters the interferogram because of the interaction between the lower resolution and the azimuth spectral shift caused by the Gaussian hill. When noise of $G=0.25$ is added to the slave, more noise enters the interferogram, which evens out the effect of the burst length.

\begin{tabular}{|c|c|c|}
\hline Noise added & Radar Mode & Phase Error \\
\hline None & Continuous & 0.0 \\
\hline None & 2-beams & 11.5 \\
\hline None & 4-beams & 13.5 \\
\hline 0.25 & Continuous & 20.0 \\
\hline 0.25 & 2-beams & 20.2 \\
\hline 0.25 & 4-beams & 21.6 \\
\hline
\end{tabular}

Table 1: Effects of Burst Cycles and noise on interferogram phase noise.

The effect of varying levels of receiver noise is further illustrated in Figure 3 for the burst lengths of 128 and 256 samples.

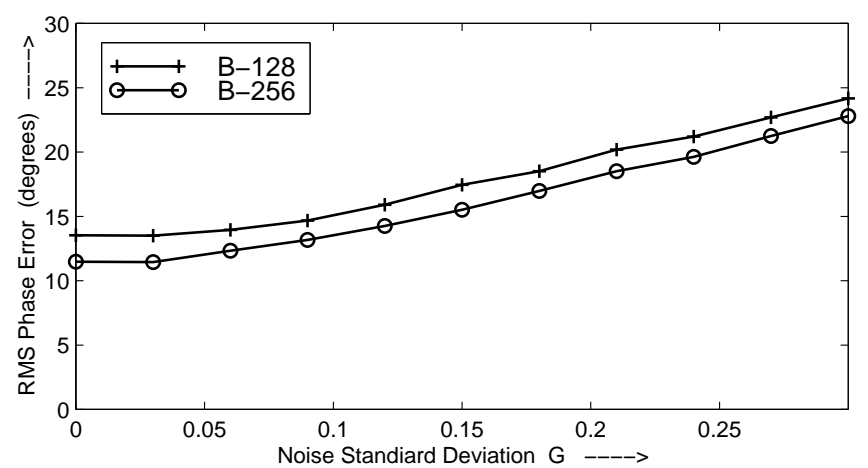

Figure 3: Effect of receiver noise and burst length on interferogram phase noise.

\section{Effect of Burst Mis-Alignment}

In this experiment, we simulate burst misalignments of up to 30 samples or about $25 \mathrm{~ms}$. This is up to $10 \%$ of the burst length in the 2-beam case, and up to $20 \%$ of the burst length in the 4-beam case. After the images are compressed, the misalignment is removed before the interferogram is made. This is feasible because InSAR processing includes fine registration steps. The results with and without receiver noise are shown in Figure 4.

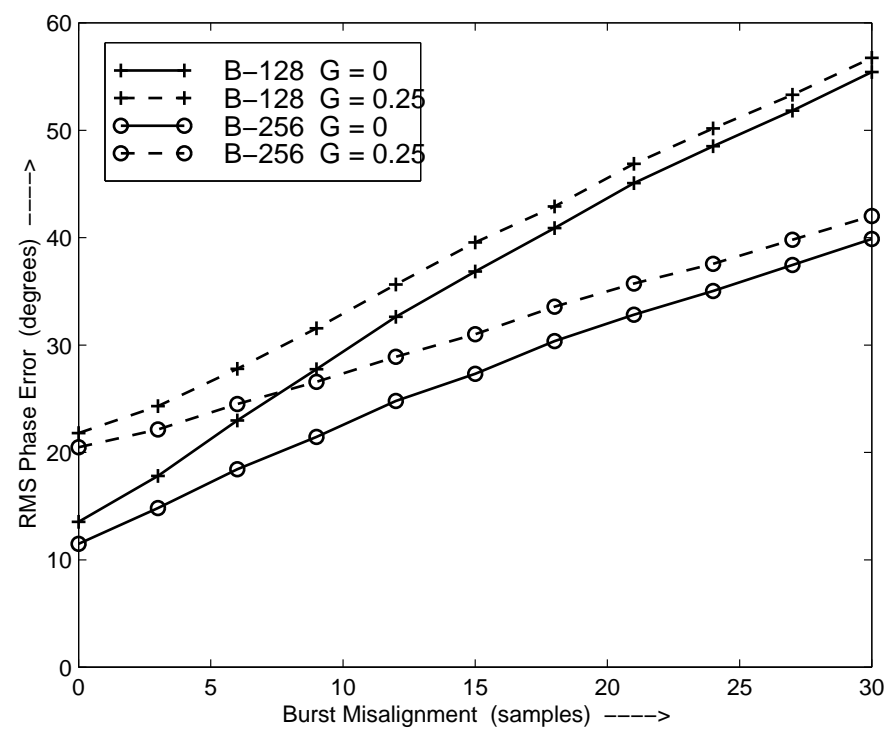

Figure 4: Interferogram phase error due to burst misalignment.

The effect of burst misalignment can be seen in the interferogram of Figure 5. The mis-alignment causes the average Doppler in the master and slave to be different, which creates uniform phase noise throughout the interferogram. In addition, where the bursts are stitched together near samples 500, 1000 and 1500, a band of complete decorrelation occurs where the master and slave have targets taken from different bursts.

As soon as the mis-registration is accurately measured, the burst-mode data can be reprocessed by windowing out the non-aligned portions of each burst. This decreases processing efficiency because the proportion of data discarded from the matched filtering operations is greater, and the IFFTs are overlapped more, but the phase noise due to misalignment will be removed. A lesser amount of phase noise will exist however, because of the coarser resolution of the processing. 


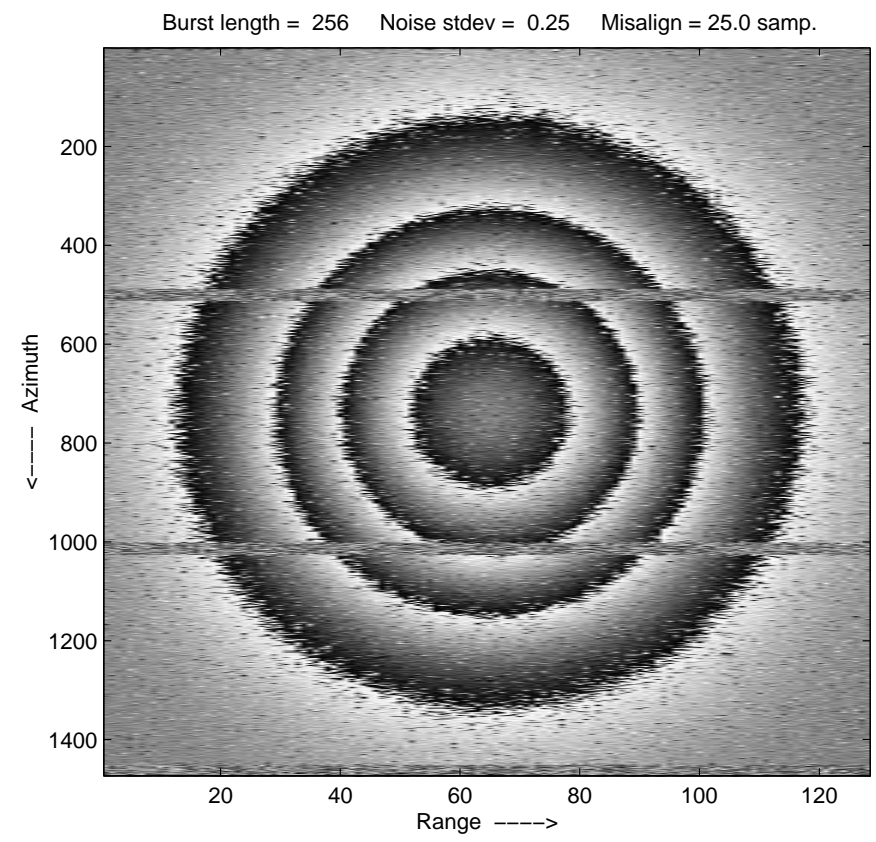

Figure 5: Interferogram phase error due to burst misalignment.

\section{Conclusions}

Using an InSAR simulation, we have seen how burstmode data has inherently lower coherence than the analogous continuous-mode data. The simulation was designed to ensure that the point spread function of each target has a phase governed by the Doppler centroid, independent of the phase of nearby targets.

The coherence is further reduced when the burst cycles are not aligned. This means that if meaningful results are to be obtained from ScanSAR operation, special care must be taken to align the bursts to a common geometric point on the ground.

\section{References}

[1] A. M. Guarnieri and C. Prati, "ScanSAR Focusing and Interferometry," IEEE Trans. Geoscience and Remote Sensing, vol. 34, pp. pp. 1029-1038, July 1996.

[2] F. Wong, D. Stevens, and I. Cumming, "PhasePreserving Processing of ScanSAR Data with a Modified Range Doppler Algorithm," in IGARSS'97, (Singapore), pp. 725-727, August 38, 1997. 